\title{
Evaluation of extreme wave probability on the basis of long-term data analysis
}

\author{
Kirill Bulgakov ${ }^{1,2}$, Vadim Kuzmin ${ }^{2}$, and Dmitry Shilov ${ }^{2}$ \\ ${ }^{1}$ Shirshov Institute of Oceanography, Russian Academy of Sciences, 36, Nahimovskiy prospekt, Moscow, 117997, Russia \\ ${ }^{2}$ Russian State Hydrometeorogical University, 98, Maloochtinsky Pr., Saint Petersburg, 195196, Russia \\ Correspondence: Kirill Bulgakov (bulgakov.kirill@gmail.com)
}

Received: 27 November 2017 - Discussion started: 16 January 2018

Revised: 6 October 2018 - Accepted: 12 October 2018 - Published: 25 October 2018

\begin{abstract}
A method of calculation of wind wave height probability based on the significant wave height probability is described (Chalikov and Bulgakov, 2017). The method can also be used for estimation of the height of extreme waves of any given cumulative probability. The application of the method on the basis of long-term model data is presented. Examples of averaged annual and seasonal fields of extreme wave heights obtained using the above method are given. Areas where extreme waves can appear are shown.
\end{abstract}

\section{Introduction}

The highest risks of economic and environmental damage for sea-based human activities, i.e. cargo shipments, fishery, oil production etc., are mostly connected with extreme weather conditions on the sea surface, among which strong storms are the foremost. It is especially difficult to predict emergency situations caused by extreme waves for those cases of seabased activities which require people's long stay at sea or prolonged use of equipment in the ocean.

One of the methods to minimize possible risks is the use of climate data based on long-term series of observations. At present there are archives consisting of reanalysis data on surface waves based on wave forecasts corrected by different methods, i.e. direct measurements using accelerometers and GPS buoys, remote measurements by satellite-borne altimetry and various types of radars. The main characteristic of wave fields included in the archive is significant wave height $H_{\mathrm{s}}$ defined as a mean value (trough to crest) of onethird of the highest of all the waves (Ochi, 1998). The value of $H_{\mathrm{s}}$ is calculated in the following way:
$H_{\mathrm{s}}=4\left(\int_{0}^{\infty} \int_{0}^{\infty} S\left(k_{x}, k_{y}\right) \mathrm{d} k_{x} \mathrm{~d} k_{y}\right)^{1 / 2}$,

where $k_{x}, k_{y}$ are wave numbers, while $S\left(k_{x}, k_{y}\right)$ is the wave spectrum.

It is evident that knowledge on significant wave height is not sufficient to evaluate real wave height for a given wave field. Extreme waves of the same height can appear with different probability for different values of $H_{\mathrm{s}}$. For example, a wave $10 \mathrm{~m}$ high can appear in both a wave field with $H_{\mathrm{s}}=10 \mathrm{~m}$ and in a wave field with $H_{\mathrm{s}}=5 \mathrm{~m}$. Or there can be waves with a height of 15 and $17 \mathrm{~m}$ in a wave field characterized by $H_{\mathrm{s}}=10 \mathrm{~m}$. Thus, $H_{\mathrm{s}}$ data do not give enough information about the probability of real wave heights.

The nature of freak waves was investigated analytically (Onorato et al., 2009) and numerically (Chalikov, 2009). Recently it was found that the statistical properties of troughto-crest wave height are quite different from those of the wave height above mean level. Studies (Chalikov and Babanin, 2016; Chalikov, 2016, 2017) show that linear and nonlinear statistics of extreme waves (defined as trough-to-crest waves) are identical not only for a broad spectrum but for one-dimensional wave fields too. This means that generation of a trough-to-crest extreme wave is the result of the simple superposition of linear modes, no matter how broad the spectrum is. This property is not found for the wave height above mean level. Thus, the statistical properties of trough-to-crest wave height can be investigated with linear modelling, just by generation of large ensembles of the superposition of linear modes with random phases and the spectrum prescribed. Thus the problem of trough-to-crest statistics becomes quite 
straightforward. Contrary to such an approach, investigation of the statistics of wave height above mean level remains a subject of non-linear wave theory. From the practical point of view, for floating objects the data on the full height (trough to crest) of a wave are more important. However, the data on probability of wave height above mean level are important for fixed-construction offshore platforms.

The theoretical probability distribution for wave crest height (or wave height above mean level) was suggested by Weibull (1951). Later it was studied on a basis of observational data in nature and wave channels (see review by Kharif et al., 2009). Extended data for estimation of probability of wave height can be obtained with integration of non-linear modes based on full equations for potential (irrotational) flow (Touboul and Kharif, 2010; Chalikov, 2009). Methods of probability calculations were considered in many papers (see, for example, Bitner-Gregersen and Toffoli, 2012; Dyachenko et al., 2016).

The most popular method of trough-to-crest wave height detection is based on a zero-crossing technique. A direct method is based on the use of moving windows; the method is applicable for both 1-D and 2-D cases.

Estimation of extreme waves today is mostly made by analysis of data of significant wave height. Jiangxia (2018), analysing long-term data, considered that an extreme wave is a wave exceeding two significant wave heights. In Larsen et al. (2015) a long-term wave dataset was analysed using the spectral method, and it was shown that the spectrum of modelled significant wave height (trough to crest) contained the energy for a frequency of more than $2.5 \times 10^{-5} \mathrm{~Hz}$ (daily timescale and less). A spectral correction method was developed to fill in the missing variability in the modelled variable at high frequencies. In Guo and Sheng (2015) the peak-over-threshold method was used to estimate the extreme significant wave heights from 30-year wave simulations. In Samayam et al. (2017) estimation of extreme wave height (crest-mean level) was made by using methods of extreme value theory. The main advantage of the method of Chalikov and Bulgakov (2017) compared with methods mentioned above is that their method is based on results of direct modelling of wave fields.

This paper is devoted to investigation of the statistics and geographical distribution of wave height above mean sea level.

\section{Description of the method}

In Chalikov and Bulgakov (2017) an algorithm for estimation of cumulative probability of waves $P(h)$ exceeding a specific value of wave height above mean level $(h)$ was developed using long-term data on $H_{\mathrm{s}}$. The description of the method is given below.

The probability of a wave exceeding a specific height $h$, if significant wave height is in a small range $\mathrm{d} H_{\mathrm{s}}$ around $H_{\mathrm{s}}$, equals $\widetilde{P}(\widetilde{H})$ for specific $\widetilde{H}=h / H_{\mathrm{s}}$ multiplied by probability of $H_{\mathrm{S}}$ in this range $\left(\widetilde{P}(\widetilde{H}) \cdot P\left(H_{\mathrm{S}}\right) \mathrm{d} H_{\mathrm{S}}\right)$, by the standard definition of conditional probability. Consequently, $P(h)$ can be determined as the integral of $\widetilde{P}(\widetilde{H}) \cdot P\left(H_{\mathrm{s}}\right)$ over all possible values of $H_{\mathrm{s}}$ :

$P(h)=\int_{0}^{H_{\mathrm{smax}}} \widetilde{P}(\widetilde{H}) P\left(H_{\mathrm{s}}\right) \mathrm{d} H_{\mathrm{s}}$,

where $P\left(H_{\mathrm{s}}\right)$ is the probability distribution of $H_{\mathrm{s}}$ for a specific point, while $H_{\mathrm{smax}}$ is the maximum value of $H_{\mathrm{S}}$ in the dataset for a specific point.

The model $H_{\mathrm{s}}$ data used for $P\left(H_{\mathrm{s}}\right)$ (Significant wave dataset calculated by WaveWatch III, 2018) were calculated with the latest version of the WaveWatch III model (Tolman, 2014) and GFS- 2 wind analysis 2 (Sasha et al., 2014). The hindcasts cover the period from August 1999 to July 2015. The spatial resolution of the dataset fields is $0.5^{\circ} \times 0.5^{\circ}$. Calibration of the model and its validation are carried out using a great number of wave buoys. The data and results of its validation are described in Chawla et al. (2013).

The approximation of $\widetilde{P}(\widetilde{H})$ was based on results of a 3-D model of potential (irrotational) flow. The model used spectral definitions of fields, finite differences for vertical derivative calculation, and a fourth-order Runge-Kutta scheme for time integration. Fourier resolution is $256 \times 64$ wave numbers, and resolution in physical space is $1024 \times 256$ (more detail in Chalikov et al., 2014). The calculations were performed for 350 units of non-dimensional time, i.e. for 70000 time steps. The initial conditions were generated on a basic JONSWAP spectrum. Model runs were calculated under the condition that input energy from wind to waves equals wave energy dissipation. This condition corresponds to fully developed wind waves. In total 75 experiments were made (more detail in Chalikov et al., 2014; Chalikov and Bulgakov, 2017).

The results of the series of experiments were processed in the following way: each wave field of surface height above mean level $(\eta)$ reproduced by the numerical model was normalized by the value of significant wave height corresponding to this field $\left(\widetilde{H}=\eta / H_{\mathrm{s}}\right)$. (Note that $\eta$ is a variable of the 3 -D model of potential (irrotational) waves. It should be distinguished from $h$ despite the fact that both $\eta$ and $h$ have the same physical sense.) Then, a non-dimensional wave field was used for the calculation of cumulative probability of nondimensional wave height $\widetilde{P}(\widetilde{H})$. The distribution obtained was approximated by the following function:

$\widetilde{P}(\widetilde{H})=\exp \left(-3.97 \widetilde{H}-4.02 \widetilde{H}^{2}\right)$.

Note that $\widetilde{P}(\widetilde{H})$ is the cumulative probability of the height of the free surface above mean level. This probability for $\widetilde{H}=1$ (the height of free surface equals significant wave height) is quite small (0.0003). 


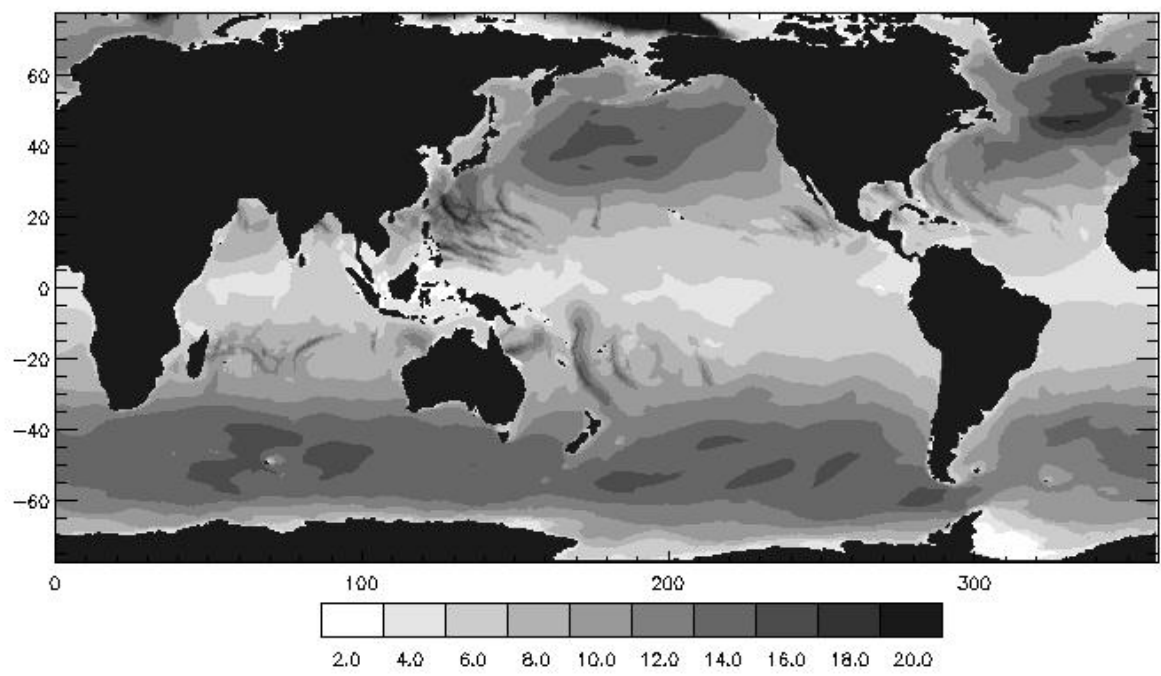

Figure 1. Wave heights $(\mathrm{m})$ above mean level with a cumulative probability of $10^{-7}$, annual average.

The above expression can be used for the interval $\leq \widetilde{H} \leq$ 1.85. The probability of a wave higher than 1.85 (the maximal value of $\widetilde{H}$ in data) can be considered extremely low and therefore is neglected. It should be noted that approximation (Eq. 3) was obtained with use of the precise 3-D model based on full non-linear equations. The volume of data used for approximation (Eq. 3) include more than 4.5 billion values of $\eta$ (number of points in a single field multiplied by the number of records in the experiment multiplied by the number of experiments). Currently, this approximation is considered universal for wind wave fields in which cases of freak waves are most likely. Waves of other types of spectrums (swells) have a small steepness and do not influence extreme wave generation except in cases in which long-wave currents can steepen shorter waves.

The spatial distribution of extreme wave probability was investigated, based on Eq. (3) from Chalikov and Bulgakov (2017) together with the spatial distribution of significant wave height from Chawla et al. (2013). In this paper results of an application of this method are considered. We show global fields of wave height with a cumulative probability of $10^{-7}$ thus calculated.

\section{Results}

Figure 1 shows an average annual field of wave heights with a cumulative probability of $10^{-7}$. It can be seen that waves with a height of up to $20 \mathrm{~m}$ above mean level can appear with such a probability, some of the extreme waves $(16 \mathrm{~m}$ and more) being found in areas of active navigation (eastern part of the Atlantic Ocean, East China Sea, Philippine Sea, Yellow Sea, south-western part of the Pacific Ocean).

The distribution of annual-average significant wave height provided by the model (Chawla et al., 2013) is shown in
Fig. 2. As seen, the maximum value in the field of annualaverage significant wave height does not exceed $5 \mathrm{~m}$ (southern area of Indian and Pacific oceans), while the height of real extreme waves can reach $16 \mathrm{~m}$ in this area. The data in Fig. 1 have a more complicated structure, due, for example, to the periods with strong wind along trajectories of tropical storms. Consequently, the calculations of the distribution of real wave height should be carried out for shorter periods, i.e. for seasonal or monthly averaged data on significant wave heights.

Figure 3 shows the field of wave height above mean level with a probability of $10^{-7}$ averaged for December-February. When comparing Fig. 3 and Fig. 1 it is seen that in midlatitudes of the Northern Hemisphere wave heights become higher. In some areas appearance of extreme wave heights exceeding $16 \mathrm{~m}$ is possible. At the same time there are actually no extreme waves in the eastern part of the Arctic Ocean, which is connected with seasonal ice formation in the area. In equatorial and tropical areas of the world ocean wave heights are lower in winter (Northern Hemisphere), compared with the average annual wave heights. It should be noted that in the western part of the Atlantic Ocean trajectories of hurricanes disappeared while the number of such trajectories increased in the Indian Ocean.

An increase in wave heights over March-May can be seen (Fig. 4) in the Southern Hemisphere. Actually all the area of mid-latitudes from the latitude of $40^{\circ} \mathrm{S}$ to the latitude of $60^{\circ} \mathrm{S}$ is characterized by a probability $>10^{-7}$ of wave heights above mean level exceeding $14 \mathrm{~m}$. In mid-latitudes of the Northern Hemisphere in spring, wave height values are more than the average annual values, though less than the winter values, while in some areas (Atlantic Ocean near Iceland, Pacific Ocean near the Bering Sea) appearance of waves exceeding $14 \mathrm{~m}$ in height above mean level is quite possible. 


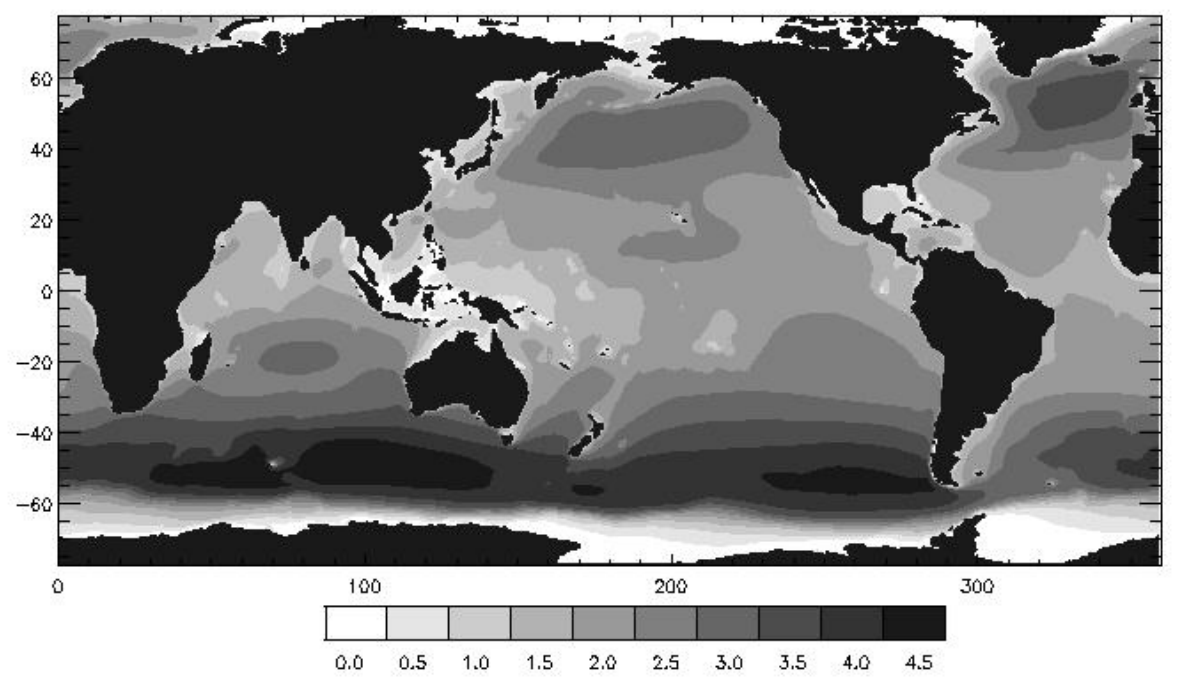

Figure 2. Average annual significant wave height (m).

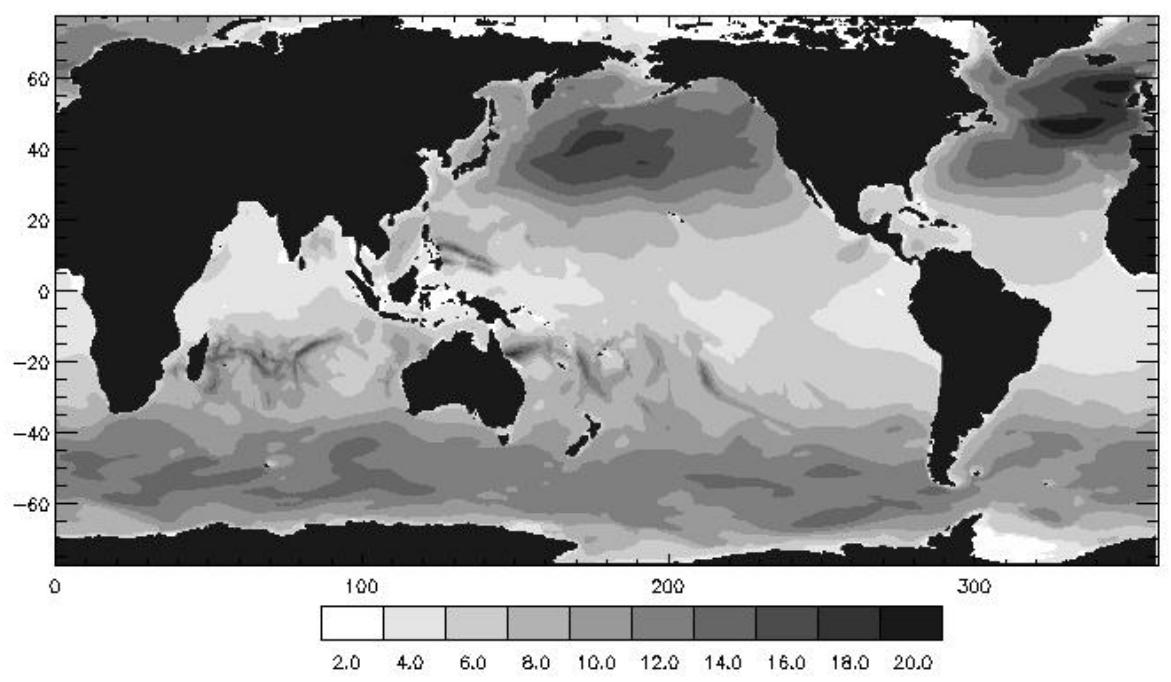

Figure 3. Wave height above mean level $(\mathrm{m})$ with a cumulative probability of $10^{-7}$ for December-February.

Summer months (Fig. 5) are characterized by a general decrease in extreme wave probability. It is especially noticeable in the northern areas of the Atlantic and Pacific oceans. Also, wave heights slightly decreased in the Southern Hemisphere. It should be noted that storm tracks appear off the eastern coast of North America and disappear in the southern part of the Pacific Ocean. In addition, quite distinct trajectories of storms appear in the eastern part of the Pacific Ocean. Small wave heights can be observed in the Arctic Ocean, in the area free from ice.

During autumn months (Fig. 6) an increase in wave heights is observed in the Arctic Ocean, with values of the extreme wave height above mean level sometimes reaching $20 \mathrm{~m}$. Among other features is an increase in the wave-free area in polar latitudes of the Southern Hemisphere, which is obviously connected with seasonal ice formation.

It is quite evident that the average monthly fields of cumulative wave height probability will allow us to obtain more exact information on the areas of extreme wave probability.

\section{Discussion and conclusions}

This paper describes a method of calculation of extreme wave probability, based on (i) wave model runs for its relation to significant wave height (Chalikov and Bulgakov, 2017) and (ii) $H_{\mathrm{s}}$ spatial distribution from 16-year hindcast data (Chawla et al., 2013). 


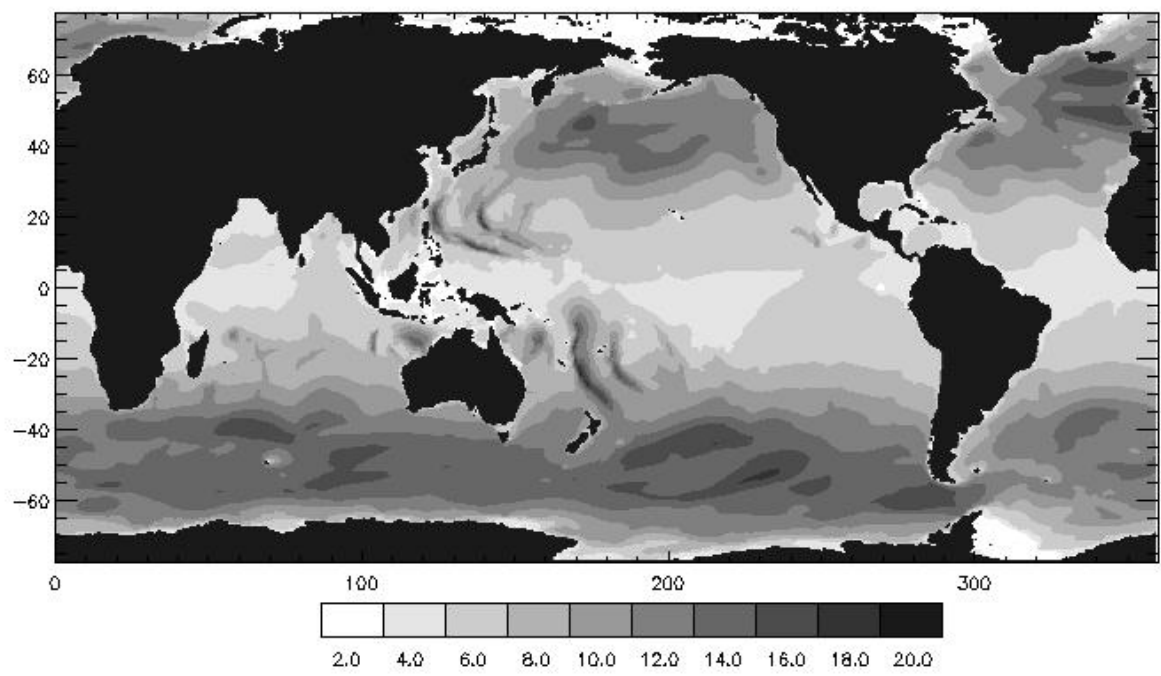

Figure 4. Wave height above mean level (m) with a cumulative probability of $10^{-7}$ averaged for March-May.

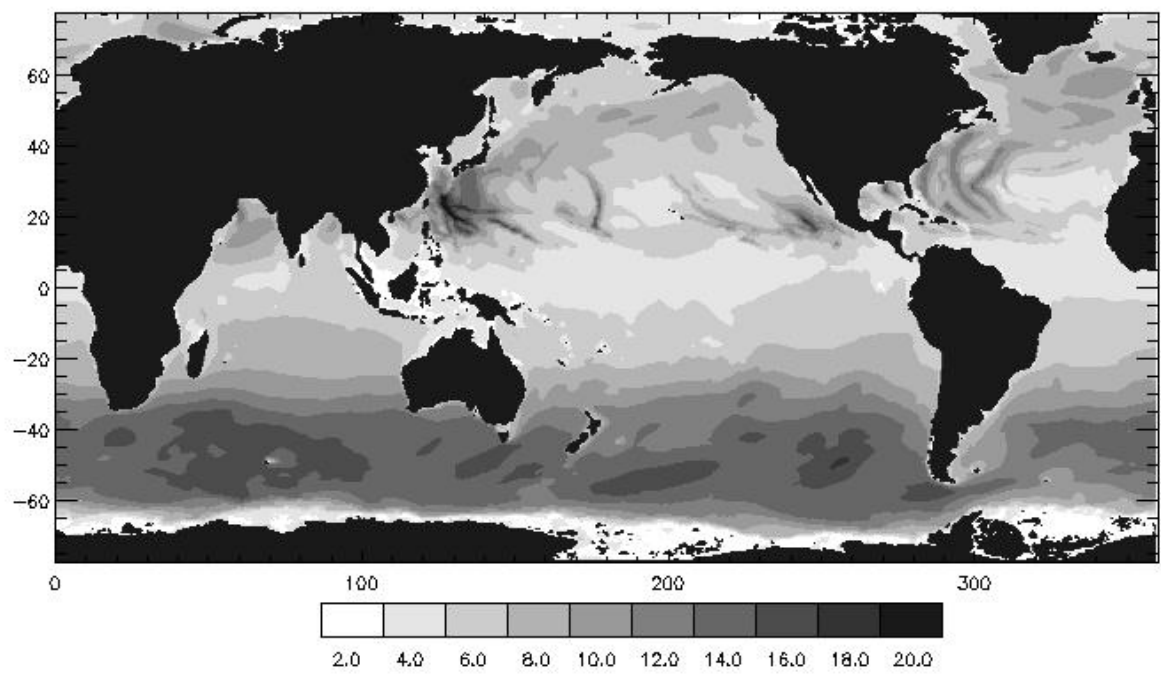

Figure 5. Wave heights above mean level (m) with a cumulative probability of $10^{-7}$, for June-August.

The method uses the results of massive numerical simulations with 3-D irrotational wave models (Chalikov et al., 2014). Initial conditions for each run were assigned by the JONSWAP spectrum, but for each run random phases were different. Such details of the initial spectrum are not too important. The ensemble modelling is used to eliminate the effects of reversible non-linear interactions causing down shifting that can influence the statistics. To be sure that the simulated process can be treated as quasi-stationary; the time of integration was chosen to be relatively short, viz. 350 units of non-dimensional time. The extensive statistics were obtained by multiple repetitions of runs with the same initial spectrum. The total number of records used for construction of approximation (Eq. 3) was 4587520000.
The wave spectrum during integration undergoes fluctuations: amplitudes grow with an increase in wave number due to reversible non-linear interactions. However, the averaged spectrum remains similar in different runs and more or less close to the spectrum assigned in initial conditions, confirming quasi-stationarity and some universality of the approximation (Eq. 3) to wave height probability. This approximation fills the gap between more or less known statistics on significant wave height and unknown statistics of real waves.

This method can be used for estimation of probability of extreme waves, which is important for designing engineering constructions. The approach here can be used to evaluate the height of waves of any given cumulative probability. It is not expedient to use values less than $10^{-9}$, which are outside the range of validity for Eq. (3) $\left(\widetilde{P}(1.85)\right.$ is approximately $\left.10^{-9}\right)$. 


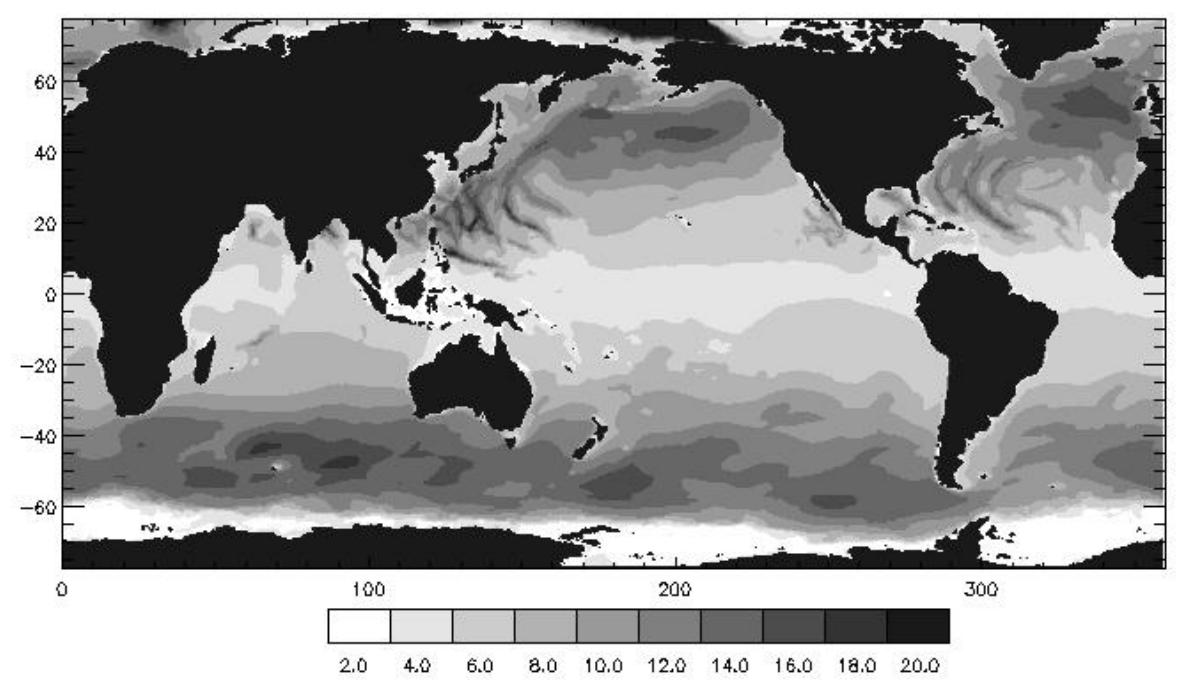

Figure 6. Wave heights above mean level $(\mathrm{m})$ with a cumulative probability of $10^{-7}$, averaged for September-November.

Hence, on the whole, the method considered is suitable for estimation of extreme values of wave heights having small probability.

The maps of the global distribution of wave heights with a probability of $10^{-7}$ for the main seasons illustrate the approach of the method. Estimation of the return period of a wave with a specific cumulative probability is quite a sophisticated problem. It will be the subject of our next work.

We do not state that results of this paper completely solve the problem of treating data on significant wave height in terms of real wave height (above mean level). The most difficult unresolved problem is the problem of estimating confidence intervals, which needs further extensive simulation and analysis.

Data availability. Data are available at https://cloud.rshu.ru/index. $\mathrm{php} / \mathrm{s} / 5 \mathrm{NoHhezK} 2 \mathrm{AEVgzL}$ (Significant wave dataset calculated by WaveWatch III, 2018).

Author contributions. KB conceived the main idea of the article, performed calculation of fields of wave height, and wrote the article. VK and DS carried out visualization of fields.

Competing interests. The authors declare that they have no conflict of interest.

Acknowledgements. The authors are thankful to Dmitry Chalikov for his useful consultations.

The investigation was fulfilled with financial support of the Russian Science Foundation (project no. 16-17-00124).
Edited by: John M. Huthnance

Reviewed by: two anonymous referees

\section{References}

Bitner-Gregersen, E. M. and Toffoli, A.: On the probability of occurrence of rogue waves, Nat. Hazards Earth Syst. Sci., 12, 751762, https://doi.org/10.5194/nhess-12-751-2012, 2012.

Chalikov, D.: Freak waves: their occurrence and probability, Phys. Fluids, 21, 076602, https://doi.org/10.1063/1.3175713, 2009.

Chalikov, D. and Babanin, A. V.: Comparison of linear and nonlinear extreme wave statistics, Acta Oceanol. Sin., 35, 99-105, https://doi.org/10.1007/313131-016-0862-5, 2016.

Chalikov, D. and Bulgakov, K.: Estimation of wave height probability based on the statistics of significant wave height, J. Ocean Eng. Mar. Energy, https://doi.org/10.1007/s40722-017-0093-7, in press, 2017.

Chalikov, D., Babanin, A., and Sanina, E.: Modeling of ThreeDimensional Fully Nonlinear Potential Periodic Waves, Ocean Dynam., 64, 1469-1486, https://doi.org/10.1007/s10236-0140755-0, 2014.

Chalikov, D. V.: Numerical modeling of sea waves, Springer, Switzerland, 330 pp., 2016.

Chalikov, D. V.: Linear and nonlinear statistics of extreme waves, Russ. J. Numer. Anal. Math. Model., 32, 91-99, 2017.

Chalikov, D. V. and Bulgakov, K. Y.: Numerical modeling of wave development under the action of wind, Phys. Wave Phenom., 25, 315-323, 2017.

Chawla, A., Spindler, D. M., and Tolman, H. L.: Validation of a thirty year wave hindcast using the climate forecast system reanalysis winds, Ocean Model., 70, 189-206, 2013.

Dyachenko, A. I., Kachulin, D. I., and Zakharov, V. E.: Probability distribution function of freak waves: nonlinear versus linear model, Stud. Appl. Math., 132, 189-198, 2016. 
Guo, L. and Sheng, J.: Statistical estimation of extreme ocean waves over the eastern Canadian shelf from 30-year numerical wave simulation, Ocean Dynam., 65, 1489-1507, 2015.

Jiangxia, L., Shunqi, P., Yongping, C., Yang-Ming, F., and Yi, P.: Numerical estimation of extreme waves and surges over the northwest Pacific Ocean, Ocean Eng., 153, 225-241, 2018.

Kharif, C., Pelinovsky, E., and Slunyaev, A.: Rogue Waves in the Ocean, in: Advances in geophysical and environmental Mechanics and Mathematics, Springer, Berlin, Germany, 2009.

Larsen, X. G., Kalogeri, C., Galanis, G., and Kallos, G.: A statistical methodology for the estimation of extreme wave conditions for offshore renewable applications, Renewable Energy, 80, 205218, 2015

Ochi, M. K.: Ocean waves: the stochastic approach, in: Cambridge Ocean Technology Series, Cambridge University Press, Cambridge, 332 pp., 1998.

Onorato, M., Waseda, T., Toffoli, A., Cavaleri, L., Gramstad, O., Janssen, P. A., Kinoshita, T., Monbaliu, J., Mori, N., Osborne, A. R., Serio, M., Stansberg, C. T., Tamura, H., and Trulsen, K.: Statistical Properties of Directional Ocean Waves: The Role of the Modulational Instability in the Formation of Extreme Events, Phys. Rev. Lett., 102, 114502, https://doi.org/10.1103/PhysRevLett.102.114502, 2009.
Samayam, S., Laface, V., Annamalaisamy, S. S., Arena, F., Vallam, S., and Gavrilovich, P. V.: Assessment of reliability of extreme wave height prediction models, Nat. Hazards Earth Syst. Sci., 17, 409-421, https://doi.org/10.5194/nhess-17-409-2017, 2017.

Sasha, S., Moorthi, S., Pan, H., and Goldberg, M.: The NCEP climate forecast reanalysis version 2, J. Climate, 27, 2185-2208, 2014.

Significant wave dataset calculated by WaveWatch III: https://cloud. rshu.ru/index.php/s/5NoHhezK2AEVgzL, last access: 23 October 2018 .

Tolman, H. L.: User manual and system documentation of WAVEWATCH III version 4.18, Technical Note 316, NOAA/NWS/NCEP/MMAB, College Park, MD, USA, 311 pp., 2014.

Touboul, J. and Kharif, C.: Two-dimensional direct numerical simulations of the dynamics of rogue waves under wind action, Adv. Numer. Simul. Nonlin. Water Waves, 11, 43-74, 2010.

Weibull, W.: A statistical distribution function of wide applicability, J. Appl. Mech., 18, 293-297, 1951. 